\title{
Desarda technique as a valuable alternative for inguinal hernia patients refusing mesh implantation: long-term results fifteen years after a pure tissue repair in 198 patients
}

\author{
Kryspin Mitura ${ }^{1,2}$, Anna Rzewuska ${ }^{3}$, Marzena Skolimowska-Rzewuska², Dorota Wyrzykowska ${ }^{1,2}$ \\ ${ }^{1}$ Faculty of Medical and Health Sciences, University of Natural Sciences and Humanities in Siedlce, Siedlce 08-110, Poland. \\ ${ }^{2}$ Department of General Surgery, Siedlce Hospital, Siedlce 08-110, Poland. \\ ${ }^{3}$ Department of Medical, Medical University in Lublin, Lublin 20-059, Poland.
}

Correspondence to: Dr. Kryspin Mitura, Faculty of Medical and Health Sciences, University of Natural Sciences and Humanities in Siedlce, 15 Starowiejska St., Siedlce 08-110, Poland. E-mail: chirurgia.siedlce@gmail.com

How to cite this article: Mitura K, Rzewuska A, Skolimowska-Rzewuska M, Wyrzykowska D. Desarda technique as a valuable alternative for inguinal hernia patients refusing mesh implantation: long-term results fifteen years after a pure tissue repair in 198 patients. Mini-invasive Surg 2021;5:22. https://dx.doi.org/10.20517/2574-1225.2021.19

Received: 9 Feb 2021 First Decision: 9 Mar 2021 Revised: 21 Mar 2021 Accepted: 29 Mar 2021 Published: 8 May 2021

Academic Editor: William W. Hope Copy Editor: Xi-Jun Chen Production Editor: Xi-Jun Chen

\begin{abstract}
Aim: The aim of the study was to retrospectively analyze long-term results of surgical treatment of patients diagnosed with primary inguinal hernia up to 15 years after a Desarda pure tissue repair.

Methods: The study was conducted on a group of adult patients with primary inguinal hernia who underwent elective surgery at our center during 2005-2006. Patients' data and hernia and surgery characteristics were recorded. Incidence of postoperative complications was assessed seven days after surgery. An attempt was made to contact all patients 15 years after the procedure regarding recurrence, possible surgical re-treatment, pain, and satisfaction.
\end{abstract}

Results: Desarda procedure was performed in 341 patients. Fifteen years after the surgical procedure, a follow-up was successful in 215 (63\%) patients, of whom 198 (58.1\%) answered all of the questions. In the early perioperative period, minor postoperative complications were found in $5.6 \%$ of patients. After 15 years of followup, three recurrences were found (1.5\%). Recurrences occurred 2, 3, and 5 years after the surgery. All patients expressed their satisfaction with the treatment. Twenty-eight patients (14.4\%) reported a rare occurrence of mild pain while performing certain activities. Three patients reported persistent chronic pain (1.5\%). 
Conclusion: Surgical repair of primary inguinal hernia using the Desarda technique is a simple, feasible, repeatable procedure, using the patient's own tissues, and with a low learning curve. It seems that the Desarda repair can still be a safe alternative to other non-mesh surgical techniques, especially when the patient refuses the use a synthetic mesh.

Keywords: Inguinal hernia, Desarda technique, pure tissue repair, recurrences, follow-up

\section{INTRODUCTION}

Inguinal hernias are a widespread pathology and every fourth man in his life will be affected by this pathology ${ }^{[1]}$. Introduction of a polypropylene mesh was a milestone in the hernia repair ${ }^{[2]}$. Currently, the most frequently used method is the Lichtenstein repair with the use of an implant strengthening the posterior wall of the inguinal canal ${ }^{[3]}$. Techniques based on the use of the patient's own tissues (pure tissue repairs) are currently losing importance due to the possibility of securing hernia with a material of known and durable strength - the polypropylene mesh ${ }^{[4]}$. Only the Shouldice method still finds supporters and the long-term results of this method used by experienced surgeons are similar to those of the Lichtenstein method $^{[5]}$. A main disadvantage of the Shouldice method is its specific degree of complexity, which means that only some surgeons perform the procedure in a fully correct manner with creation of all four layers of reconstructed tissues ${ }^{[3,5]}$.

An alternative to both of these methods was proposed in 2001 by an Indian surgeon - Desarda ${ }^{[6]}$. He described a method of repair which took into account the biomechanical aspect of the inguinal canal ${ }^{[6,7]}$. A bilaterally pedunculated strip of the external oblique aponeurosis is used to strengthen the posterior wall of the inguinal canal [Figure 1]. As a consequence, and contrary to the Shouldice method, tissues of the transverse fascia, which were initially weakened due to a disturbed collagen structure, are not used ${ }^{[7]}$. The strip is not separated at both its poles, which that it is included in the dynamic system of forces and stresses occurring in this area. Tension of the abdominal muscles causes the strip of aponeurosis of the external oblique muscle of the abdomen to tighten and expand, simultaneously pressing the posterior wall of the inguinal canal and strengthening the area of the deep ring ${ }^{[7]}$.

The aim of the study was to retrospectively analyze long-term results of surgical treatment of patients diagnosed with primary inguinal hernia up to 15 years after a Desarda repair surgery. An additional aim of this work is to discuss technical aspects of the presented procedure in detail.

\section{METHODS}

\section{Patients}

The study was conducted on a group of adult patients with primary inguinal hernia who underwent elective surgery at our center during 2005-2006. All patients operated during this period were qualified for the Desarda repair procedure on the basis of typical indications for the surgical treatment of inguinal hernias, in accordance with current surgical guidelines. Only elective procedures were included in the analysis. Patients with recurrent and strangulated hernias were excluded from the study. It was assumed that patients in whom the external oblique aponeurosis was observed intraoperatively to be significantly thinned or separated into fibers were not eligible for the Desarda surgery, but during the 2-year observation period no patients were excluded for this reason. Surgical procedures were performed by the same team of surgeons. Patients were operated on under regional anesthesia or, in the event of contraindications to this type of anesthesia, under general anesthesia. All patients received thromboprophylaxis $12 \mathrm{~h}$ prior to surgery (enoxaparin). All patients were preventively treated with a prophylactic dose of an antibiotic 30 min prior to 

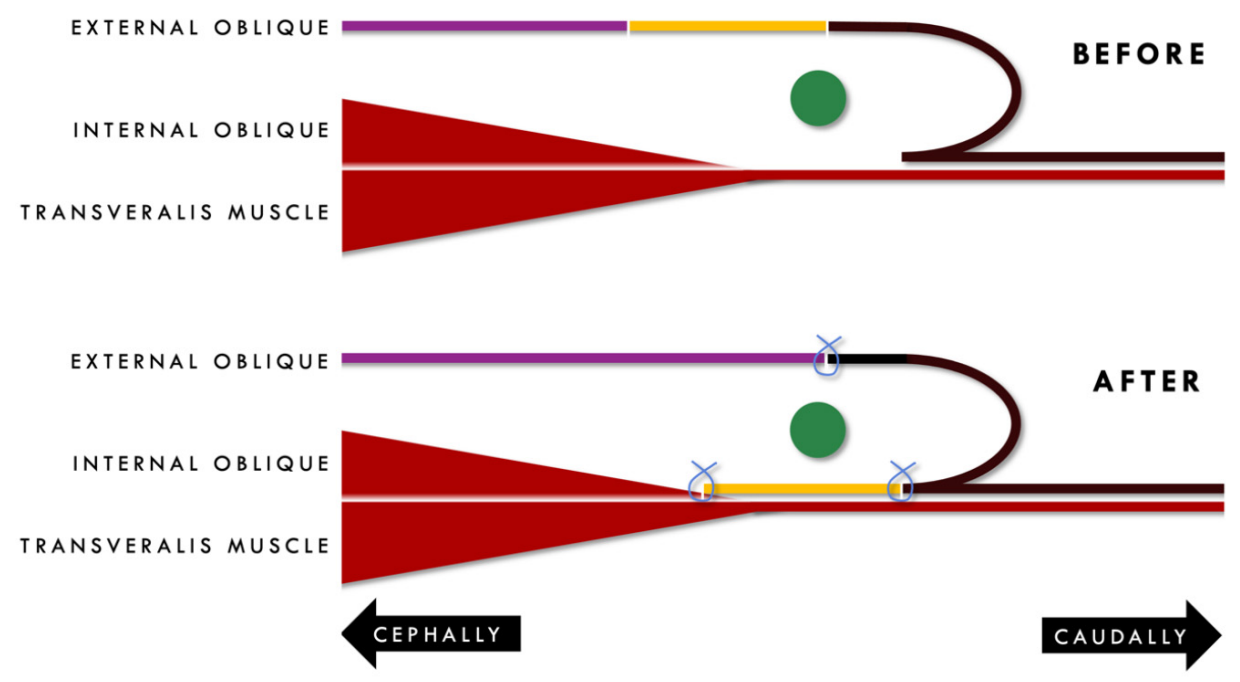

Figure 1. A cross-section scheme of the inguinal canal demonstrating the principles of Desarda repair: a narrow strip of external oblique aponeurosis (yellow) is moved onto a posterior wall of inguinal canal behind spermatic cord (green) and sutured to both the inguinal ligament and the internal oblique (blue). The anterior wall of the inguinal canal is closed with upper/medial flap of the external oblique aponeurosis (purple).

first incision (cefazolin). Patients were enrolled in the study after obtaining their informed consent to participate in the study.

\section{Data and characteristics}

Data regarding anthropometric parameters during the surgery (age, sex, and BMI), ASA classification, hernia characteristics (side, location, orifice diameter, and conversion of the hernia type/size to the currently used EHS classification), and duration of the surgical procedure were recorded ${ }^{[3]}$. In the immediate postoperative period (up to seven days), incidence of postoperative complications such as hematoma, wound infection, seroma, and testicular swelling was assessed in the operated patients. An attempt was made to contact all patients who underwent Desarda surgery 15 years after the date of the procedure. A team surgeon performed a telephone survey with all available patients. Contacted patients answered four questions from the questionnaire during a telephone interview. The questions concerned recurrence, possible surgical re-treatment of the hernia on the same side, pain at rest and during exercise (according to the Visual Analog Scale), and satisfaction with the treatment result.

\section{Procedure}

The skin is incised at a typical site, about $2 \mathrm{~cm}$ above and parallel to the inguinal ligament, over a distance of about 5-6 cm. Subcutaneous tissue is incised, paying attention to subcutaneous veins in this area and ligating them. Coagulation of these vessels is avoided as they can be a source of extensive postoperative hematomas. After cutting the Scarpa fascia and dissection of the deep subcutaneous tissue, the aponeurosis of the external oblique abdominal muscle becomes visible. Its condition is visually controlled: thickness, strength, and separation into individual fibers. An apparently thinned structure and the aponeurosis separated into fibers disqualifies from the use of a Desarda method. Sometimes there is a slight rupture in the aponeurosis due to a passage of nerves. These can be used later to create a strip. The superficial inguinal ring is exposed. At its apex, the aponeurosis lamellae are incised parallel to the course of fibers, opening the inguinal canal [Figure 2A] (to clearly visualize the structures of the inguinal canal, the following figures show the anatomical situation of the inguinal region in a female patient). The spermatic cord (or round ligament of the uterus in females) is isolated together with the hernial sac from the external oblique 

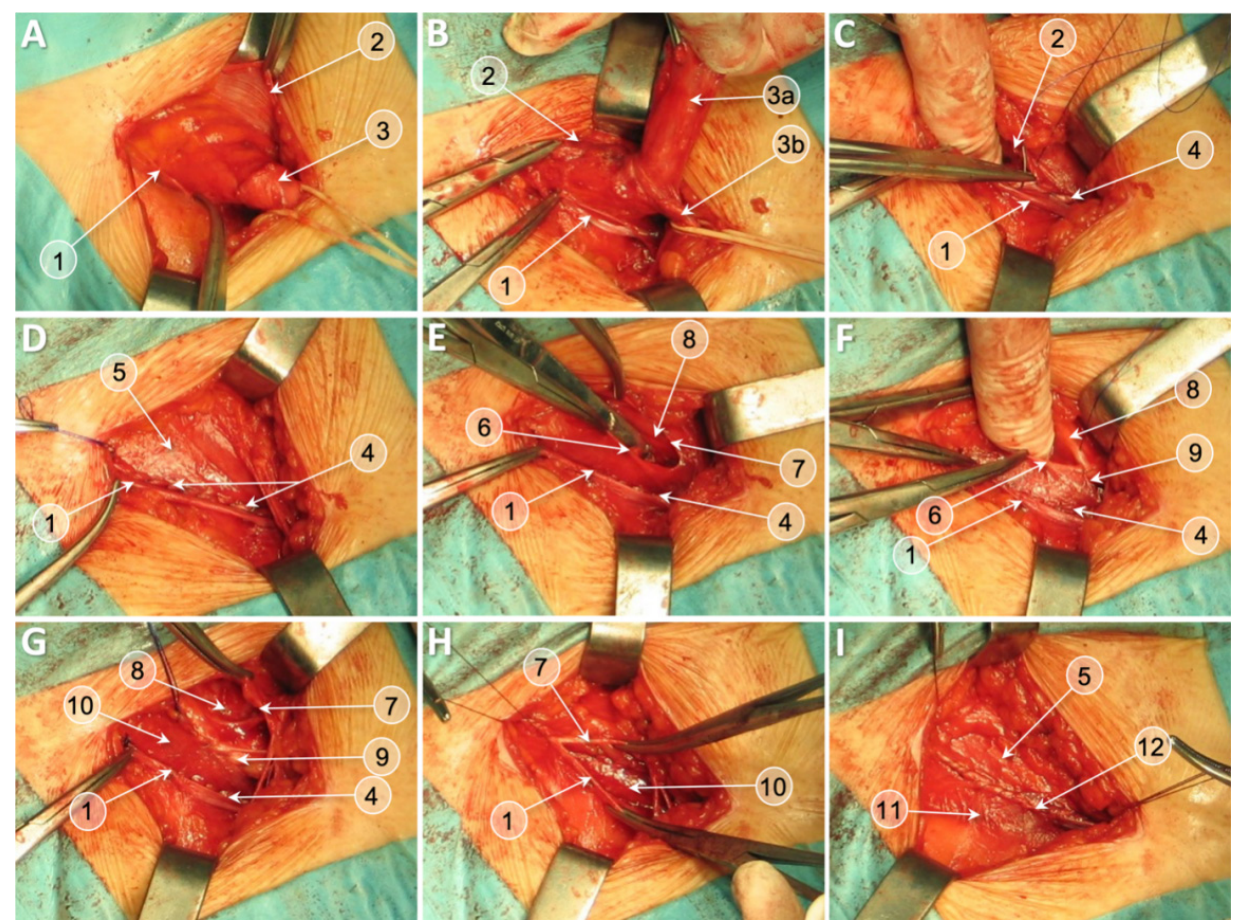

Figure 2. Desarda repair of a groin hernia in female patient (with round ligament resection for better visualization). Anterior wall of inguinal canal is opened (A); hernia sac is dissected (B); lower edge of medial/upper flap of external oblique is sutured to inguinal ligament ( $C, D)$; creation of external oblique strip with longitudinal incision $(E)$; the strip is sutured to internal oblique $(F, G)$; and the anterior wall of inguinal canal is closed with external oblique (H, I). Inguinal ligament (1); upper/medial flap of external oblique (2); undissected hernia sac [(3a) dissected hernia sac; and (3b) round ligamentum of uterus] (3); suture line between lower edge of upper/medial flap of external oblique and inguinal ligament (4); external oblique aponeurosis (5); upper edge of a created aponeurotic strip (6); lower edge of the upper flap of remaining incised external aponeurosis (7); internal oblique muscle (8); suture line between upper edge of the strip and internal oblique (9); completed and sutured on both edges aponeurotic strip reinforcing inguinal floor (10); lower flap of initially incised external oblique aponeurosis attached to inguinal ligament (11); and suture line of anterior wall of inguinal canal (12).

aponeurosis. The ilioinguinal, iliohypogastric nerves should be identified, and the genital branch of the genitofemoral nerve should be identified to prevent damage to these structures. The iliohypogastric nerve becomes visible after the separation of the lamina of the internal oblique muscle. The genital nerve can be relatively easily protected by visualizing the veins on the posterior side of the spermatic cord while it is being released from the bottom of the inguinal canal. Avoiding damage to the superficial veins of the spermatic cord protects the genital nerve that runs in this area. The isolated spermatic cord/round ligament is retracted using a rubber drain [Figure $2 \mathrm{~B}$ ]. The hernia sac is managed in the usual manner. After dissecting it to the neck area, the unopened sac is usually pushed into the abdominal cavity. If there are doubts as to the contents of the sac, it should be opened and then sutured and ligated, after checking the content, and the excess is cut off.

In the next stage, the actual hernioplasty is performed using the Desarda technique [Figure $2 \mathrm{C}$ and $\mathrm{D}$ ]. Both flaps of the external oblique aponeurosis (medial/superior and lateral/inferior) are visualized and dissected. Using a continuous single-fiber non-absorbable 2.0 suture (Surgipro ${ }^{\circ}$, Tyco), the lower edge of the medial (superior) flap of the external oblique aponeurosis is sutured to the shelving margin of inguinal ligament under the spermatic cord, from the pubic tubercule up to the level of the deep ring. In this way, the deep inguinal ring is recreated, as in the Lichtenstein method - so that it passes freely on the tip of the finger. Excessive constriction of the spermatic cord should be avoided. Then, a $2-2.5 \mathrm{~cm}$ wide aponeurosis strip is 
made, cutting parallel to the course of fascia fibers, parallel to the inguinal ligament, passing through a possible rupture in the aponeurosis (the place where the iliohypogastric nerve passes). In this way, an aponeurotic strip and a medial flap of the external oblique aponeurosis are formed [Figure $2 \mathrm{E}$ ]. The medial aponeurotic flap is later used to close the inguinal canal. In the next step, the medial edge of a newly formed lateral aponeurotic strip is secured with a continuous suture to the underlying internal oblique abdominal muscle, using the same material as above [Figure $2 \mathrm{~F}$ ]. In this way, an aponeurotic strip is obtained which is attached to the inguinal ligament on one side and to the internal oblique abdominal muscle on the other side. It extends upward to the distal part of the aponeurosis and downwardly attaches in the region of the upper ramus of the pubic bone [Figure $2 \mathrm{G}$ ]. This strip strengthens the posterior wall of the inguinal canal, similar to the polypropylene mesh in the Lichtenstein method. The inguinal canal is then closed by suturing the remaining flaps of the fascia with absorbable suture over the spermatic cord/round ligament, typically reconstructing the superficial inguinal ring [Figure $2 \mathrm{H}$ and I]. Single stitches on the subcutaneous tissue and the skin complete the procedure.

\section{Statistical analysis}

The results obtained during the study were subjected to basic statistical analysis in Microsoft Excel 16.45. Descriptive analysis included the calculation of average values, standard deviations, and proportions. Bivariate analysis was done using Pearson's Chi-square and Fisher's exact test for categorical variables as applicable, and $t$ test for continuous variables. A value of $P<0.05$ was considered a statistically significant difference between the compared groups. All calculations were performed using the Statistica 13.0 licensed statistical analysis software package.

\section{RESULTS}

In total, the elective repair according to a Desarda procedure was performed in 341 patients. Fifteen years after the surgical procedure, a phone call follow-up was successful in 215 (63\%) patients, of whom 198 (58.1\%) answered all of the questions. The characteristics of treated patients and the procedure performed are summarized in Tables 1 and 2. There were no relevant statistical differences between the respondents and the total population of operated on patients. In the early perioperative period, minor postoperative complications were found in $5.6 \%$ of patients, all of which resolved spontaneously without additional surgical intervention. After 15 years of follow-up, three recurrences were found, which constituted $1.5 \%$ of the patients who answered the questions [Table 3]. Recurrences occurred 2, 3, and 5 years after the treatment, respectively. Nevertheless, all patients expressed their satisfaction with the treatment. Twentyeight patients (14.4\%) reported a rare occurrence of mild pain while performing certain activities, but at the same time they emphasized that this is not a phenomenon that hindered their everyday functioning. Three patients reported persistent chronic pain (1.5\%).

\section{DISCUSSION}

There is a common consensus that, after Lichtenstein repair procedures, the percentage of observed complications and the number of recurrences are similar for procedures performed by both experienced surgeons and residents ${ }^{[8]}$. This is largely the result of a short learning curve characteristic for the procedure. Simplicity of this operation favors the speed of its performance and similar results are achieved both by centers specializing in hernia surgery and small surgical departments. A similar situation seems to be the case with the Desarda surgery. Many common features between these two techniques (i.e., exactly the same anatomical structures need to be dissected in both methods) lead to the conclusion that, as with the Lichtenstein surgery, the Desarda method is lacking a troublesome complexity. Usually, duration of the surgical procedure reflects the scale of difficulties of individual surgical techniques in inguinal hernia repairs. The lack of statistically significant differences in the duration of procedures using the Desarda and 
Table 1. Demographic details of all patients and in patients with 15-year follow-up

\begin{tabular}{|c|c|c|c|}
\hline & All patients & Patients in follow-up & $P$ \\
\hline Patients, $n(\%)$ & $341(100 \%)$ & $198(58.1 \%)$ & \\
\hline Age, mean (SD), years & $51.9(14.3)$ & $51.4(15.1)$ & 0.186 \\
\hline \multicolumn{4}{|l|}{ Gender, n (\%) } \\
\hline Male & $306(89.7 \%)$ & $178(89.9 \%)$ & 0.183 \\
\hline Female & $35(11.3 \%)$ & $20(11.1 \%)$ & 0.204 \\
\hline $\mathrm{BMI}$, mean (SD), $\mathrm{kg} / \mathrm{m}^{2}$ & $25.9(2.8)$ & $25.8(2.6)$ & 0.293 \\
\hline \multicolumn{4}{|l|}{ ASA } \\
\hline I & $191(56.0 \%)$ & $117(59.1 \%)$ & 0.072 \\
\hline ॥ & $125(36.7 \%)$ & $69(34.8 \%)$ & 0.108 \\
\hline III & $25(7.3 \%)$ & $12(6.1 \%)$ & 0.116 \\
\hline \multicolumn{4}{|l|}{ Smoking, $n(\%)$} \\
\hline Yes & $201(58.9 \%)$ & $119(60.1 \%)$ & 0.091 \\
\hline No & $140(41.1 \%)$ & $79(39.9 \%)$ & 0.162 \\
\hline \multicolumn{4}{|l|}{ Hernia reducibility, $n$ (\%) } \\
\hline Yes & $317(93.0 \%)$ & $182(91.9 \%)$ & 0.137 \\
\hline No & $24(7.0 \%)$ & $16(8.1 \%)$ & 0.171 \\
\hline
\end{tabular}

Table 2. Characteristics of procedures in all patients and in patients with 15-year follow-up

\begin{tabular}{|c|c|c|c|}
\hline & All patients & Patients in follow-up & $P$ \\
\hline Procedures, $n(\%)$ & $341(100 \%)$ & $198(58.1 \%)$ & \\
\hline \multicolumn{4}{|l|}{ Anesthesia, $n$ (\%) } \\
\hline Spinal & $293(85.9 \%)$ & $172(86.9 \%)$ & 0.426 \\
\hline General & $48(14.1 \%)$ & $26(13.1 \%)$ & 0.318 \\
\hline \multicolumn{4}{|l|}{ Hernia type, $n$} \\
\hline Direct (M1/M2/M3) & $109(37 / 25 / 47)$ & $63(24 / 12 / 27)$ & 0.227 \\
\hline Indirect (L1/L2/L3) & $232(81 / 67 / 84)$ & $135(45 / 44 / 46)$ & 0.108 \\
\hline \multicolumn{4}{|l|}{ Surgeon, $n(\%)$} \\
\hline Resident & $88(25.8 \%)$ & $48(24.3 \%)$ & 0.517 \\
\hline Attending surgeon & $253(74.2 \%)$ & $150(75.7 \%)$ & 0.330 \\
\hline Operation time, mean (SD), min & $51.4(16.2)$ & $52.5(17.3)$ & 0.199 \\
\hline \multicolumn{4}{|l|}{ Nerve resection, $n(\%)$} \\
\hline Iliohypogastric & $74(21.7 \%)$ & $45(22.7 \%)$ & 0.243 \\
\hline Ilioinguinal & $7(2.1 \%)$ & $4(2.1 \%)$ & 0.302 \\
\hline Genital branch of femoral & $0(0.0 \%)$ & $0(0.0 \%)$ & \\
\hline
\end{tabular}

Lichtenstein techniques may indicate their similar degree of difficulty ${ }^{[8,9]}$. This distinguishes the Desarda surgery from other low-tension techniques, especially the Shouldice technique, where it has been documented that satisfactory surgical treatment results (recurrence rate below 1\%) are obtained only in specialized centers and after operations performed by surgeons experienced in Shouldice technique ${ }^{[10]}$. Bracale et al. ${ }^{[11]}$ in a meta-analysis of fourteen randomized controlled trials revealed that Shouldice repair lasted 7 min longer than Lichtenstein repair, while Desarda repair was 6 min shorter than the average Lichtenstein procedure.

Various methods of surgical treatment of inguinal hernia are being compared mainly in terms of recurrence rate. In the analyzed group, 15 years after the surgical procedure, only three recurrences were found. In the literature, the incidence of recurrences after Lichtenstein surgery does not exceed 1\%. Kockerling pointed 


\begin{tabular}{|c|c|}
\hline & Patients in follow-up \\
\hline & $n=198$ \\
\hline Early postoperative complications & $11(5.6 \%)$ \\
\hline Hematoma & $5(2.5 \%)$ \\
\hline Surgical site infection & $0(0 \%)$ \\
\hline Seroma & $4(2.0 \%)$ \\
\hline Scrotal/testicular oedema & $2(1.0 \%)$ \\
\hline Pain (VAS), mean (SD) & $0.44(0.41)$ \\
\hline \multicolumn{2}{|l|}{ Verbal description of pain, $n(\%)$} \\
\hline No pain & $167(84.4 \%)$ \\
\hline Mild pain & $27(13.6 \%)$ \\
\hline Moderate pain & $4(2.0 \%)$ \\
\hline Severe pain & $0(0 \%)$ \\
\hline \multicolumn{2}{|l|}{ Pain occurrence, $n(\%)$} \\
\hline No pain & $167(84.4 \%)$ \\
\hline Incidental & $28(14.1 \%)$ \\
\hline Constant pain & $3(1.5 \%)$ \\
\hline Foreign body sensation, $n(\%)$ & $1(0.5 \%)$ \\
\hline Loss or change of sensation, $n(\%)$ & $43(21.7 \%)$ \\
\hline \multicolumn{2}{|l|}{ Patient's full satisfaction, $n(\%)$} \\
\hline Yes & $198(100 \%)$ \\
\hline No & $0(0 \%)$ \\
\hline \multicolumn{2}{|l|}{ Recurrence, $n(\%)$} \\
\hline Yes & $3(1.5 \%)$ \\
\hline No & $195(98.5 \%)$ \\
\hline
\end{tabular}

out that the recurrence rate after inguinal hernia repairs can still be as high as $11 \%$, of which $43 \%$ appear even 10 years after the initial surgery ${ }^{[12]}$. There are limited data on the long-term incidence of this complication after the Desarda surgery. Among 1320 inguinal hernia repairs performed by the author of the discussed technique, with at least 7 years of follow-up, only one early recurrence was found, which was a result of an error during surgery ${ }^{[6,7]}$. At that time, a direct hernia was identified by mistake, leaving the coexisting indirect hernia unrecognized and, therefore, not properly managed. Szopinski et al.$^{[9]}$ reported two recurrences in a group of 105 patients after Desarda repair (1.9\%) which occurred in the weakened area of the posterior wall or internal inguinal ring up to 3 years after the surgery. These results were confirmed by Bracale et al. ${ }^{[11]}$ in a recent systematic review pointing out that the results of Desarda repair have a low recurrence rate similar to the one after the Lichtenstein technique. It seems that these optimistic data will change with popularization of the method and the treatment of a larger number of patients, but results achieved so far should be considered satisfactory.

The undoubted advantage of the Desarda method is the simplicity of its implementation, even by surgeons with little experience in hernia surgery. This brings the learning curve of this technique closer to the way of gaining experience in the Lichtenstein operations. Inguinal hernia repairs are among the most frequently performed operations in surgical departments, especially by young surgeons. It seems that development of hernia techniques should take into account specific needs of this group, i.e., allow the repair to be simple, safe, easy to learn and perform, without the risk of more serious complications during and after the procedure, and with a minimum number of recurrences ${ }^{[13]}$. Both the Desarda and Lichtenstein techniques meet the criteria described. Therefore, the Desarda surgery may successfully compete with other open 
approach repairs, both with mesh and the use of patient's tissues only.

The Desarda repair may be successfully used in all patients with a normal external oblique aponeurosis. It is especially recommended in cases of surgeries on young slim men with lateral hernias with a narrow internal ring $^{[14]}$. The procedure meets all conditions of modern hernia repair: it can be performed on a single-day basis, it is possible to mobilize the patient early, patients may resume professional activities soon after the procedure, only minimal pain is reported, there are no serious complications and recurrences, and a low learning curve is observed ${ }^{[3,15]}$.

In some cases, this technique is not recommended in patients in whom the external oblique aponeurosis is thinned and separating into fibers. The Lichtenstein surgery is preferred in those cases. Despite certain technical limitations, the Desarda surgery seems to be a certain alternative allowing for hernia repair without the use of mesh implants, especially with regards to the growing number of patients refusing use of mesh. However, there are regions with limited access to modern mesh repairs, mainly in low-income countries, where an effective and safe pure tissue repair may be the only accessible alternative to worldwide recommended mesh repairs ${ }^{[16]}$. However, there are many issues that have not been answered yet. Will longterm results of these repairs be similar to, or perhaps surpass, those performed using the Lichtenstein method? Will congenital defects in collagen structure, which are mentioned as the cause of hernia pathogenesis, affect long-term results of this treatment ${ }^{[17]}$ ? The long-term follow-up results presented above supplement the knowledge available in this field. However, the weakness of the study is a low percentage of patients who were available for the long-term follow-up. Taking into account the 15-year follow-up period, it seems that gathering more patients may be impossible, unless the results are entered into dedicated central national medical registers.

There are several limitations to the present study. This study was a retrospective review of a single center's experience. Although it is possible that recurrence results could have been biased from progressive variations, such as alterations in surgeon's experience or other unaccounted for recognized practices, this would be uncertain given surgeon workforce constancy, unchanged surgical practice, and established postoperative pathways throughout the study time phase. Furthermore, patient's sex and age, BMI, smoking habit, and hernia type and size (the major determinants that might influence a recurrence rate) were all similar between the patients included in the follow-up and the entire group of operated on patients. Another possible limitation is the lack of personal examination. Although it was possible to invite some of the patients for a face-to-face interview along with sonography examination, most. Patients were unwilling to undertake a visit, making it unlikely to analyze the outcomes in a large group of patients. A third limitation of our study was that the cohort was not compared with the results in a group treated with a standardized technique (i.e., Lichtenstein repair). This likely reflects the fact that, during the initial implementation of this technique in our center, Desarda repair was a predominant surgical technique used for primary groin hernia repair in the analyzed time period. Despite the retrospective and descriptive character of the study as its drawback, the main intention of the authors was to familiarize the readers with the details of the surgical technique and to present long-term treatment results.

In conclusion, surgical repair of primary inguinal hernia using the Desarda technique is a simple, feasible, repeatable procedure, using the patient's own tissues, and with a low learning curve. The results of treatment of inguinal hernias using this technique allow for a low percentage of recurrences and chronic pain in a long-term follow-up. It seems that the Desarda repair can still be a safe alternative to other nonmesh surgical techniques, especially when the patient refuses the use a synthetic mesh. 


\section{DECLARATIONS}

\section{Authors' contributions}

Conception and design of the study: Mitura K, Skolimowska-Rzewuska M

Data analysis: Mitura K, Skolimowska-Rzewuska M, Wyrzykowska D

Interpretation: Mitura K, Skolimowska-Rzewuska M

Data acquisition: Mitura K, Rzewuska A, Wyrzykowska D

Administrative, technical, and material support: Mitura K, Rzewuska A, Wyrzykowska D

\section{Availability of data and materials}

Data supporting these findings are deposited in Siedlce hospital records.

Summarized tables and questionnaires are deposited in Prof. Mitura's academic repositories.

\section{Financial support and sponsorship}

None.

\section{Conflicts of interest}

All authors declared that there are no conflicts of interest.

\section{Ethical approval and consent to participate}

The study has been designed and performed in accordance with the Declaration of Helsinki and approved by a Bioethics Committee of the Regional Medical Chamber in Warsaw, Poland (reference number: $\mathrm{OIL} / \mathrm{KB} / 12 / 2005)$. An informed consent was obtained from all participants.

\section{Consent for publication}

Not applicable.

\section{Copyright}

(c) The Author(s) 2021.

\section{REFERENCES}

1. Primatesta P, Goldacre MJ. Inguinal hernia repair: incidence of elective and emergency surgery, readmission and mortality. Int $J$ Epidemiol 1996;25:835-9. DOI PubMed

2. Lichtenstein IL, Shulman AG, Amid PK, Montllor MM. The tension-free hernioplasty. Am J Surg 1989;157:188-93. DOI PubMed

3. Mitura K, Garnysz K, Michałek I. Long-term follow-up of a randomized controlled trial of Lichtenstein repair vs the Valenti technique for inguinal hernia. Hernia 2019;23:547-54. DOI PubMed PMC

4. Lorenz R, Arlt G, Conze J, et al. Shouldice standard 2020: review of the current literature and results of an international consensus meeting. Hernia 2021. DOI PubMed

5. Desarda MP. Inguinal herniorrhaphy with an undetached strip of external oblique aponeurosis: a new approach used in 400 patients. Eur J Surg 2001;167:443-8. DOI PubMed

6. Desarda MP. Physiological repair of inguinal hernia: a new technique (study of 860 patients). Hernia 2006;10:143-6. DOI PubMed

7. Group. International guidelines for groin hernia management. Hernia 2018;22:1-165. DOI PubMed PMC

8. Amid PK. Lichtenstein tension-free hernioplasty: its inception, evolution, and principles. Hernia 2004;8:1-7. DOI PubMed

9. Szopinski J, Dabrowiecki S, Pierscinski S, Jackowski M, Jaworski M, Szuflet Z. Desarda versus Lichtenstein technique for primary inguinal hernia treatment: 3-year results of a randomized clinical trial. World J Surg 2012;36:984-92. DOI PubMed PMC

10. Lichtenstein IL, Shulman AG, Amid PK. The Cause, prevention, and treatment of recurrent groin hernia. Surg Clin North Am 1993;73:529-44. DOI PubMed

11. Bracale U, Melillo P, Piaggio D, et al. Is Shouldice the best NON-MESH inguinal hernia repair technique? Int J Surg 2019;62:12-21. DOI PubMed

12. Köckerling F, Simons MP. Current Concepts of Inguinal Hernia Repair. Visc Med 2018;34:145-50. DOI PubMed PMC

13. Mitura K, Garnysz K, Wyrzykowska D, Michałek I. The change in groin pain perception after transabdominal preperitoneal inguinal hernia repair with glue fixation: a prospective trial of a single surgeon's experience. Surg Endosc 2018;32:4284-9. DOI PubMed PMC

14. Köckerling F, Koch A, Adolf D, et al. Has Shouldice repair in a selected group of patients with inguinal hernia comparable results to Lichtenstein, TEP and TAPP techniques? World J Surg 2018;42:2001-10. DOI PubMed PMC 
15. Mitura K, Śmietański M, Kozieł S, Garnysz K, Michałek I. Factors influencing inguinal hernia symptoms and preoperative evaluation of symptoms by patients: results of a prospective study including 1647 patients. Hernia 2018;22:585-91. DOI PubMed PMC

16. Mitura K, Kozieł S, Pasierbek M. Groin hernia surgery in northern Ghana--humanitarian mission of Polish surgeons in Tamale. Pol Przegl Chir 2015;87:16-21. DOI PubMed

17. Öberg S, Andresen K, Rosenberg J. Etiology of inguinal hernias: A comprehensive review. Front Surg 2017;4:52. DOI PubMed PMC 